\title{
TRADUIRE EN NORVÉGIEN DES CONSTRUCTIONS DÉTACHÉES FRANÇAISES
}

\author{
NELLY FOUCHER STENKLØV ET EIRIK HVIDSTEN \\ Université norvégienne de sciences et de technologie
}

RÉSUMÉ

Le présent article consiste en une étude de la construction détachée en français au prisme de sa traduction en norvégien par des apprenants de niveau B1. Plus précisément, nous nous pencherons sur les cas de syntagmes adjectivaux et participiaux en constructions détachées. En inscrivant leur description théorique dans le cadre formel de Combettes $(1998,2005)$, nous nous attarderons sur deux aspects de ces structures - le repérage référentiel et la relation sémantique - autant de potentiels défis à la compréhension de l'apprenant.

Une enquête établie en deux temps (tests 1 et 2) constituera ensuite l'empirie de nos analyses. Dans une démarche de recherche-action, à partir des descriptions théoriques fournies, nous dresserons un inventaire des écueils syntaxiques et sémantiques sur lesquels se heurtent les étudiants dans leurs traductions. Ce répertoriage mettra en exergue les différences d'emplois fondamentales entre le norvégien et le français et nous guidera vers une conclusion contrastive didactique à l'intention des apprenants.

\section{[1] INTRODUCTION}

En français comme en norvégien, le syntagme en position initiale de l'énoncé occupe un rôle discursif essentiel, soit parce qu'il assure une connexion, soit parce qu'il annonce un nouveau thème. Toutefois, chacune des langues a ses propres contraintes syntaxiques qui empêchent une reproduction conforme de l'ordre et de la nature des syntagmes. Ce clivage entre l'intention discursive commune aux deux langues et leurs impératifs syntaxiques divergents constitue un grand défi d'apprentissage du français L2 dont nous proposons d'éclairer quelques pans en consacrant le présent article à certains types de constructions détachées (CD) en zone initiale dans les énoncés. Plus précisément, nous porterons notre attention sur les difficultés propres au traitement syntaxico-sémantique et, conséquemment, à la compréhension de certains usages de la CD en français par les apprenants norvégiens.

La $\mathrm{CD}$, souvent assimilée à la grande famille des appositions, est une prédication syntaxiquement réduite et implicitement attachée à la phrase matrice : 
(1) a. Stoppée net par la pandémie, la vie culturelle suffoque.

b. Enfin, parsemés, il y avait des cactus.

Limitant notre champ d'étude aux syntagmes adjectivaux et participiaux antéposés à la phrase matrice, nous tablerons sur les traductions norvégiennes d'une dizaine d'étudiants en français L2. Entre autres problèmes rencontrés, deux se dégageront, concernant d'une part la question de l'identification référentielle, d'autre part celle de la relation sémantique. Nous inscrivant dans le cadre descriptif formel de Combettes $(1998,2005)$, nous préconiserons avec Helland (2013) une démarche de repérage référentiel et envisagerons certains aspects de l'interface syntaxico-sémantique susceptibles d'influer sur l'acceptabilité d'occurrences en termes de référence. Enfin, nous nous pencherons sur les modes de connexions entre prédications impliqués par les syntagmes adjectivaux et participiaux en $\mathrm{CD}$, sur leur impact dans le traitement cognitif de la référence et sur la façon dont les apprenants norvégiens les interprètent.

\section{[2] DESCRIPTION SÉmANTICO-PRAgMATIQUE DE LA CD}

Les $\mathrm{CD}$ sont marquées par une variation et complexité considérable. En nous basant sur Combettes (2005: 33), nous considérons les constructions suivantes comme faisant partie des CD : les constructions absolues (avec sujet exprimé), les infinitifs prépositionnels, le gérondif, les participes, les adjectifs et les syntagmes nominaux sans déterminant.

\section{[2.1] La structure des CD}

Formellement, en contraste avec les autres $C D$, les constructions absolues sont marquées par un sujet exprimé. Nous prenons ici comme point de départ la définition étendue du terme « construction absolue ». Dans la définition stricte de Combettes $(1998,2005)$, seul (2b) constitue une construction absolue, pourtant, quelques approches y incluent également les constructions participiales, approche que nous adoptons ici (voir Hobæk Haff, 2012). Regardons quelques exemples :

a. La porte fermée, Marie est partie en courant.

b. Son arme sur la table de nuit, il s'est enfin endormi.

c. Ce phénomène étant un des plus grands problèmes pour la théorie, il faudra y consacrer encore plus de recherche.

Puisque le sujet est exprimé dans ces constructions, en contraste avec les constructions que nous traiterons plus bas, il n'y a pas d'ambiguïté au niveau de l'identité du sujet. Le sujet dépend d'un prédicat logique, représenté par le 
participe fermé en (2a) et les attributs sur la table de nuit et un des plus grands problèmes pour la théorie dans $(2 \mathrm{~b}-\mathrm{c})$, respectivement. Le sujet ne peut pas être coréférentiel avec le sujet de la phrase superordonnée :

(3) *La fenêtre (une fois) ouverte, elle se mit à grincer (Combettes, 2005 : 33).

Cependant, les autres $C D,{ }^{1}$ comme par exemple les constructions participiales standard et le gérondif, ${ }^{2}$ 'ont pas de sujet exprimé. L'absence de sujet exprimé entraîne une nouvelle difficulté : acceptant la prémisse selon laquelle toute phrase a un sujet, exprimé ou non, on suppose que toute phrase contient un sujet 'sous-entendu'. Dans ces constructions, ce sujet doit être établi par d'autres moyens. En grammaire générative, il est établi par la présence d'un pronom nul, (grand) PRO, normalement contrôlé par un élément de la phrase matrice (Chomsky, 1981), ou d'un (petit) pro dans les approches récentes. Prenons l'exemple suivant comme point de départ :

(4) $\left[\mathrm{PRO}_{\mathrm{i}}\right.$ Sortant dans la rue], $\mathrm{Paul}_{\mathrm{i}}$ a rencontré Jeanne.

Intuitivement, un locuteur français identifierait le sujet de sortir comme Paul. On suppose donc l'existence d'un PRO à la place du sujet de sortir.

Viennent ensuite les deux derniers types de CD : les syntagmes adjectivaux et les syntagmes nominaux :

a. Heureuse, elle est sortie rencontrer son mari.

b. Chef de l'entreprise, il a décidé de licensier trois personnes.

Il existe peu de recherches sur ce type de constructions en syntaxe formelle. Le travail de Helland \& Hobæk Haff (2000) contient un bon aperçu des défis liés à l'analyse de ces constructions. Comme on peut le constater en (6a), l'adjectif heureuse modifie le syntagme (pro-)nominal elle, pourtant, il ne se trouve pas à l'intérieur de ce dernier. Comme Helland \& Hobæk Haff le soulignent, ces

[1] Comme mentionné par Combettes (2005 : 33), les infinitifs prépositionnels peuvent également constituer un type de $\mathrm{CD}$, vu qu'ils sont souvent en position initiale de la phrase, et manquent de sujet exprimé en tant que forme infinie du verbe :

(i)

Après avoir fait des devoirs, Paul est sorti jouer au foot avec ses amis.

[2] Le gérondif est une construction apparentée au participe présent (Halmøy, 2003 ; Helland, 2013). Cette construction comporte un subordonnant en en et manifeste des propriétés similaires mais également différentes du participe présent :

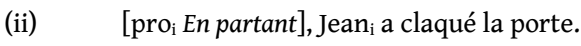

Puisqu'un traitement du gérondif dépasserait le cadre du présent article, nous en ferons abstraction ici. 
constructions constituent un défi pour les analyses syntaxiques car il est difficile de définir la fonction syntaxique des éléments en question. Qu'ils soient considérés comme des attributs libres, des appositions, des éléments subordonnés au syntagme nominal ou des adverbiaux, il est néanmoins important pour toute analyse syntaxique d'établir un lien ou une relation dans la structure de base entre le constituant détaché et le constituant qu'il modifie dans la phrase matrice.

Comme on peut le constater en regardant les exemples plus haut, les CD participiales sont marquées par une défectivité syntaxique et sémantique. Au niveau syntaxique, cette défectivité réside dans le fait qu'elles manquent d'éléments nécessaires pour former une phrase complète. Elles sont privées de verbe fini, propriété inhérente des phrases complètes et indépendantes du type déclaratif, impératif et interrogatif. Comme nous pouvons le constater à partir de la hiérarchie présentée plus haut, la plupart des CD manquent également de sujet exprimé, contrairement aux phrases déclaratives et interrogatives. Cette défectivité syntaxique des $C D$ porte également sur leur sémantique. L'absence de verbe fini les rend incapables d'ancrer le sens qu'elles véhiculent dans le temps pour former une proposition complète vérifiable par des conditions de vérité. Pour cette raison, ces constructions dépendent non seulement syntaxiquement, mais également sémantico-pragmatiquement des phrases superordonnées auxquelles elles sont rattachées. Pourtant, en français, ce dernier lien peut souvent être sousspécifié, permettant plusieurs interprétations possibles. Ce fait est également en vigueur pour les $\mathrm{CD}$ du type adjectival et nominal, représenté en (5). Nous expliciterons cette problématique dans la section suivante.

\section{[2.2] La contribution discursive}

Mettant en scène le gérondif, les exemples de Halmøy mettaient en évidence l'importance du contexte discursif pour cette saisie du contrôle du sujet. De fait, toute tentative de séparation des considérations syntaxiques et sémantico-pragmatiques devient artificielle voire inappropriée à la compréhension de la $\mathrm{CD}$, gérondif ou autre. Envisageant avec Combettes une définition de la CD comme cadre discours où elle conjugue deux propriétés cruciales dans le bon fonctionnement discursif : « lien avec l'amont, portée vers l'aval » (2005:36), on pourra plus aisément expliquer les questions de référence. Considérons les exemples suivants, comme autant d'illustrations de facettes de ce phénomène :

(6) a. Plus jeune, il lui aurait été impossible de pratiquer le yoga convenablement.

b. Autour d'elle, tombé sans doute de ses épaules, on voyait un manteau goudronné (Combettes, $1998: 146)$. 
c. Impatiente ou non, nous ne sauterons pas dans le premier avion pour rendre visite à ta mère.

Les exemples ci-dessus ne tiennent pas strictement du contexte oral. Ce dernier ne justifie donc pas à lui seul le non-respect des règles normatives de co-indexation des sujets. Dans le cas de (6c), on relèvera la désambiguïsation du référent du groupe adjectival impatiente ou non par le jeu des accords. "Impatiente » réfère obligatoirement au seul nom féminin, ta mère.

Pour expliquer des cas similaires à (6a) (où plus jeune qualifie lui) et (6b) (renvoyant tombé à son référent manteau), ou encore (6c) (où ta mère est impatiente ou non), Combettes suggère par ailleurs qu'on examine ces syntagmes adjectivaux et participiaux en $C D$ au prisme des relations circonstancielles qu'ils établissent, non pas comme de réels circonstants cadratifs - syntagmes prépositionnels, subordonnées circonstancielles - qui portent des marques claires de leur rôle, mais pour la valeur circonstancielle qu'ils induisent implicitement tout en établissant stylistiquement une continuité du thème porté par un référent déjà activé dans le discours : on sait ainsi à qui réfère ce pronom lui en (6a). Dans cette optique, on notera que la juxtaposition de la CD à un circonstant cadratif (autour d'elle) en (6b) consolide l'idée que la non co-référentialité des sujets est facilitée dès lors que la CD participe à l'expression circonstancielle (de lieu) dans l'énoncé. Parallèlement, les $C D$ de $(6 a)$ et $(6 c)$ révèlent les valeurs circonstancielles respectives de temps et de concession (Combettes, 2005).

Nous reviendrons dans notre analyse sur la part de confusion que cette prédication sur un autre argument que le sujet risque d'engendrer, notamment chez des apprenants L2. Dans l'immédiat, ce dernier commentaire sur l'expression circonstancielle nous invite à nous pencher sur les divers bagages sémantiques portés par les syntagmes adjectivaux et participiaux en CD.

\section{[2.3] Bagage sémantico-pragmatique de la CD}

Dans sa description, Combettes distingue trois variations discursives de CD : les $\mathrm{CD}$ descriptives, les constructions apparentées aux « cadres localisateurs et temporels » et les constructions opérant des « liens [discursifs] logiques». Pour ce, il examine la portée de leur prédication. Par souci d'efficacité et d'adéquation avec les objectifs de la présente étude, nous insérerons la catégorisation de Combettes dans une approche plus sémantique des liens entretenus entre la CD et la phrase matrice. Une telle description débouche sur deux types de relations : la relation descriptive et la relation circonstancielle.

La construction strictement descriptive a une relation attributive avec l'élément de la phrase matrice auquel elle réfère et sur lequel sa portée sémantique est concentrée. Elle dérive ainsi naturellement de prédications simples : Il est 
sorti furieux./Il est sorti, furieux./Furieux, il est sorti. Il est alors souligné qu'un verbe exprimant l'apparition ou la localisation du référent favorise le maintien de la valeur attributive. (2005 : 37) Dans le cadre de notre démarche empirique, quelques rares entrées du test correspondent à cette définition : Diplômée et resplendissante, Jeanne s'est levée pour faire un discours. / Reconstruite dans un style moderne, l'école est au centre du village.

Dans cette même catégorie, nous choisirons d'insérer le cas des apostrophes (Jeunes gens, écoutez-bien les paroles sages de ce vieil homme!) pour les limites de leur portée sémantique et leur sens descriptif, bien qu'elles soient laissées pour compte dans l'inventaire de Combettes, puisqu'elles ne résultent pas syntaxiquement de la dérivation décrite plus haut.

Enfin, la valeur attributive résulte d'une interprétation parmi d'autres comme l'illustre l'exemple suivant, issu de notre test :

Tout jeune garçon, Pierre était calme.

On y verra effectivement une possibilité d'interprétation descriptive, avec une portée de tout jeune garçon restreinte au nom propre Pierre: Pierre était un jeune garçon calme. Parallèlement, on considérera la variante interprétative circonstancielle de temps dont la portée sémantique s'étend cette fois sur toute la prédication première : Quand il était jeune garçon, Pierre était calme.

Ceci nous amène à présenter l'autre pendant sémantique des $\mathrm{CD}$. Si dans son optique discursive, Combettes différencie les cadres localisateurs et temporels des liens logiques, nous choisirons pour notre part de les regrouper pour le bagage circonstanciel qu'ils véhiculent tous. Combettes le précise, contrairement aux participes, un adjectif véhiculant une valeur de condition a une portée réduite aux limites de la phrase, de façon assez semblable aux CD à sens descriptif comme dans l'exemple Chaude, cette soupe ferait du bien aux randonneurs. Or, nos intentions d'analyse portent sur les formes de traduction sélectionnées dans tel ou tel cas de CD. Il nous importera dès lors davantage de savoir si certains apprenants ont décelé la nature conditionnelle de la prédication chaude que de nous assurer qu'ils ont compris que chaude a une portée relativement limitée à cette soupe.

Ainsi, nous regrouperons dans une seule catégorie les $C D$ exprimant des valeurs circonstancielles en établissant une simple dichotomie entre les valeurs qui seront traitées dans notre analyse, le cadre temporel d'une part, les liens logiques de cause ou de condition d'autre part.

Comme le panel de français consultés dans notre enquête a pu le confirmer, les $C D$ françaises peuvent aisément prendre des valeurs circonstancielles. Aleksandrova $(2015: 76)$ relève à ce sujet que le groupe nominal en CD peut esquisser 
une relation circonstancielle. L'exemple déjà cité en (7) montre que l'observation de Aleksandrova peut être appliquée au cas des syntagmes participiaux et adjectivaux, mettant en exergue la complexité à comprendre et traduire le « sémantisme propre » de l'adjectif dans le syntagme tout jeune synonyme du syntagme nominal de l'exemple. Havu \& Pierrard (2018) renchérissent dans un travail de comparaison entre les subordonnées circonstancielles et les constructions participiales détachées. Ils soulignent les différences de mode d'élaboration de ces deux formes et rapportent qu'en raison des manques et absences affectant les prédications dans le cas des emplois de $\mathrm{CD}$, les lectures générées peuvent être plurielles. (2018:11)

On soulignera enfin quelques facteurs mentionnés par Combettes (2005 : 3940) brouillant les pistes interprétatives. D'une part, l'usage de verbes présentatifs semble restreindre la portée de la CD aux éléments 'présentés' :

Et puis, parsemés, il y avait des cactus.

D'autre part, nombreuses sont les utilisations de CD où le participe et le complément de lieu associés définissent un cadre aussi temporel que spatial, tout en suggérant une délimitation de leur portée au seul co-référent dans la phrase matrice. L'interprétation flotte ainsi entre le circonstanciel de temps, le circonstanciel de lieu et le descriptif, comme dans l'exemple suivant :

(9) Tout juste arrivés dans la propriété madrilène, les enfants se jetèrent dans la piscine.

Si notre test est dépourvu de telles entrées, on verra toutefois que les types de constructions présentés engendrent une série d'ambiguïtés interprétatives auxquelles les apprenants parent en ayant recours à des outils linguistiques pas toujours appropriés. L'examen de la portée sémantique - et de ses limites - crucial pour différencier une description d'un cadrage circonstanciel, ne semble pas faire partie de l'arsenal interprétatif des étudiants B1.

\section{[2.4] La CD en norvégien}

Suite à la discussion de la section précédente, nous porterons ici notre attention sur les possibles traductions des CD françaises en norvégien. Les syntagmes adjectivaux et participiaux en $\mathrm{CD}$ sont rares en norvégien. Pour cette raison, et comme notre test à venir l'illustrera, la traduction directe n'est pas possible.

A ce point de notre étude, la manière la plus adéquate de rendre compte des limitations syntaxico-sémantiques de la CD en norvégien est contrastive. En effet, on constate à partir des entrées de nos tests que seuls trois cas de CD sont 
susceptibles d'être traduits par des constructions qui s'en rapprochent en occupant la position initiale de l'énoncé :

a. Plus jeune, Dimitri avait peu confiance en lui. $\rightarrow$ Som ung hadde Dimitri lav selvtillit.

b. Tout jeune garçon parmi les grands, Pierre n'avait pas souvent le droit de décider dans la cour de récréation.

$\rightarrow$ Som helt fersk blant de store guttene hadde ikke Pierre ofte muligheten til å bestemme ting i skolegården.

Seul le syntagme prépositionnel introduit par «som » (approximativement traduit par " en tant que ») peut assurer un respect conforme de l'ordre des mots de la phrase française. "Som », toutefois, impose des contraintes liées à l'interface syntaxico-sémantique. Là où som ung et som helt fersk... sont parfaitement acceptables, le jugement syntaxique se heurtera à des som yngre ou som varm proposés par les étudiants du test 2. Sans nous attarder sur la complexité de " som », nous rapporterons seulement qu'il semble plus approprié en association avec des adjectifs qualifiant l'état d'êtres animés à un moment de leur vie. Ceci exclut tout comparatif et tout qualificatif d'états fluctuants que nous n'aborderons pas dans cette étude, mais que notre analyse nous donnera l'occasion d'effleurer.

a. Diplômée ou non, nous offrons à Jeanne un poste intéressant dans notre entreprise.

$\rightarrow$ Ferdigutdannet eller ikke, vi tilbyr Jeanne en interessant stilling $\mathrm{i}$ selskapet vårt.

b. Enfant ou adulte, chacun à sa manière pourra apprécier le spectacle.

$\rightarrow$ Voksne som barn, alle skal kunne sette pris på forestillingen.

La construction ferdigutdannet eller ikke semble être une phrase elliptique du type " om hun er ferdigutdannet eller ikke ». Il s'agit ici probablement de deux phrases indépendantes et non pas d'une CD. Nous donnons deux raisons pour cette explication : l'ordre V2 n'est pas imposé et il y a une pause marquée entre l'élément initial et la phrase qui suit. Il en va de même pour l'exemple (11b).

(12) Diplômée et resplendissante, Jeanne s'est levée pour faire un discours.

$\rightarrow$ Ferdigutdannet og slående vakker reiste Jeanne seg for å holde en tale.

Un contexte officiel, solennel ou poétique peut faciliter l'usage de la CD dont la portée descriptive se limite au sujet de la phrase matrice.

En norvégien, l'antéposition d'un syntagme adjectival entrave l'établissement 
d'un lien sémantico-pragmatique entre la CD et la phrase matrice. La phrase, devient inacceptable. En l'absence des stratagèmes énumérés ci-dessus, le syntagme adjectival ou participial en $\mathrm{CD}$, si fréquent en français, cède sa place à des alternatives plus explicites en norvégien.

\section{[3] ENQUÊTE}

\section{[3.1] Description méthodologique}

La démarche adoptée dans la présente étude prend ancrage dans les travaux méthodologiques sur l'évaluation des compétences linguistiques en FLE (Demeuse et al. 2005). Elle s'apparente globalement à la méthode empirico-inductive. Les prédictions qui nourrissent nos réflexions ont émergé, dans un premier temps, d'observations et relevés de commentaires d'étudiants en situation de cours de traduction. Grosso modo, nous en restions sur le constat que le traitement de la CD échappait au balisage didactique du module de traduction alors qu'il s'imposait par exemple dans la presse quotidienne de la France et de la francophonie. Les apprenants ne sont certes pas armés pour produire en français des CD. Plus grave encore : ils ne le sont pas toujours non plus pour comprendre le contenu sémantique des énoncés recelant ces $C D$.

Nos problématiques balbutiées sur la base de ces premières remarques nous ont ainsi orientés vers un besoin de tests et nous ont permis d'en mettre au point les items. Si, par le biais des tests, la collecte de données relève d'une méthode hypothético-déductive afin de mieux cerner les enjeux intrinsèques aux prédictions encore intuitivement définies, les cohortes consultées - trois enseignants francophones et neuf apprenants norvégiens - sont beaucoup trop petites pour prêter aux statistiques.

A mi-chemin entre le qualitatif et le quantitatif, notre investigation s'est ainsi établie naturellement en un processus didactique de recherche-action (Puren, 2013) sur trois étapes. Un premier repérage des difficultés à interpréter les CD a généré quelques prédictions (i) à la base des tests 1 et 2 élaborés et soumis sous deux formes distinctes à deux cohortes (ii). Notre analyse a pris sa forme finale lors d'une séance de retours sur les résultats aux tests auprès des cohortes concernées (iii). ${ }^{3}$

[3] Dans tous les résultats d'enquête, les phrases du test seront données telles qu'elles sont référencées dans l'annexe et le numéro qui leur correspond apparaîtra après la phrase. Ce numéro, en italique, est donné entre accolades. Les traductions norvégiennes sont référencées ainsi : le numéro indique à quelle phrase du texte la traduction correspond. La lettre qui suit fait référence à l'étudiant. L'exemple est rendu dans sa version originale et nous faisons le choix d'ignorer toute faute d'expression qui n'a pas directement affaire à la CD. 


\section{[3.2] Prédictions}

Posant au préalable que les CD qui gênent le plus les apprenants L2 sont celles qui n'ont pas de sujet exprimé, nous avons restreint le champ d'étude aux cas des syntagmes adjectivaux et participiaux, avec, à quelques exceptions près, certains items recelant des syntagmes nominaux incluant un adjectif porteur du bagage sémantique. On associera ainsi un syntagme nominal du type tout jeune garçon au syntagme adjectival correspondant tout jeune, considérant les deux pareillement, en raison de leur rôle sémantique dans l'énoncé. Il en est de même pour le syntagme enfant ou adulte que nous considérons pour son bagage qualificatif.

Partant de là, trois prédictions ont motivé nos choix d'items dans le test de traduction :

- P1: L'ordre des mots et la forme de la traduction norvégienne calquent ceux de la phrase source française, même lorsque l'usage norvégien prescrit de s'en détacher.

- P2 : Le choix prioritaire d'interprétation du lien sémantique entre la CD et la phrase matrice est celui de la connexion descriptive

- P3 : Le fait que la CD ne soit pas «contrôlée » par le sujet de la phrase matrice complique la saisie de l'énoncé.

Les items donnés en annexe constituent, dans un autre ordre parfaitement aléatoire, le test auxquels neuf apprenants norvégiens évalués à un niveau B1 acquis ont été soumis en novembre 2020. Hormis les items distracteurs $(28,29$ et 30), chaque entrée est chevillée à, au moins, l'une des deux dernières prédictions annoncées plus haut, en plus de la première.

Le processus d'identification du sujet a été expliqué dans notre introduction théorique. En termes de prédication, on envisage sans peine un effort de traitement plus important lorsque la syntaxe d'un énoncé met en jeu l'opération de co-indexation entre le sujet vide de la CD et celui de la phrase matrice (Helland, $2006 / 2014)$. On supposera ainsi que les items $\{15\}$, $\{19\}$ et $\{27\}$ présentent des difficultés de traitement liées à l'identification du sujet de la $\mathrm{CD}$. $\{15\}$ met en scène une prédication sur l'objet direct. La prédication de $\{19\}$ porte pour sa part sur un pronom indéfini sujet (" chacun») que nous jugeons moins explicite qu'un simple groupe nominal. Enfin, en raison de l'impératif, le sujet à identifier pour la CD de $\{27\}$ n'est pas exprimé dans la phrase matrice.

La deuxième orientation de notre exploration concerne la nature de la connexion sémantique entre l'énoncé de la phrase matrice et celui de la CD. Nous avons signalé dans notre présentation théorique contrastive quelques limites syntaxiques au recours aux CD en norvégien. Par ailleurs, reprenant la description de Combettes $(1998,2005)$, nous avons mis en évidence la différence entre 
le rapport descriptif et le rapport circonstanciel qu'une $\mathrm{CD}$ peut entretenir avec l'élément de la phrase matrice auquel elle réfère. Dans la foulée des travaux de Havu \& Pierrard (2018: 11), nous postulons que le non-dit inhérent à la CD engendre diverses lectures. Or, le bagage circonstanciel, plus subjectif, moins neutre que le bagage descriptif est probablement plus difficile à cerner pour un apprenant L2. Cette problématique sera illustrée par les items $\{1\}$ à $\{27\}$. Pour cette série, dans le cadre du test 1 , trois français ont été invités à identifier la nature de la relation entretenue entre la CD et la phrase matrice. Nous reviendrons sur le détail des réponses. Dans l'immédiat, notons simplement que $\{1\}$ à $\{22\}$ expriment selon eux une relation circonstancielle tandis que $\{23\}$ à $\{27\}$ s'en tiennent à une relation descriptive. On souligne par ailleurs le cas particulier de $\{3\}$, qui, selon la cohorte consultée dans le test 1 , pourra être interprété différemment selon qu'on y voit une relation descriptive (Pierre était un tout jeune garçon calme) ou une relation circonstancielle (Quand il était jeune, Pierre était un garçon calme).

Une comparaison des résultats de cette consultation en amont du test 2 aux traductions des étudiants nous permettra d'affiner nos prédictions en formulant quelques questions supplémentaires qui tiendront lieu de jalons de notre analyse: Quelles sont les structures syntaxiques que les étudiants ont privilégiées dans les traductions des CD ? Quels sont les tenants, mais surtout les aboutissants syntaxiques et sémantiques de ces choix de structures? Enfin, pour répondre aux besoins de notre méthode action-recherche en assurant un retour didactique auprès des apprenants de notre étude, peut-on dégager une hiérarchie qualitative en termes syntaxico-sémantiques des types de traductions des syntagmes adjectivaux et participiaux en structures détachées?

\section{[3.3] Présentation des tests}

La série d'items tenant lieu de test (1 et 2) est en annexe. Afin de donner toute liberté d'interprétation aux neuf apprenants norvégiens L2, de niveau B1 acquis, la seule consigne donnée pour le test 2 était formulée ainsi : "Traduire les phrases suivantes en norvégien. Souvenez-vous : dans le doute, on ne traduit pas les mots, on traduit le sens. »

$\mathrm{Au}$ vu des résultats de traduction obtenus, nous avons décidé ensuite de soumettre une autre cohorte - trois enseignants français - à un test 1 tenant lieu de référence pour le test 2 sur l'expression des relations sémantiques. Il s'agissait là d'établir un point de départ relativement objectif concernant l'interprétation du sens des rapports créés entre les phrases matrices et leurs CD dans les items du test 2. La consigne aux Français était formulée ainsi : "Pour chaque entrée cidessous, indiquer le sens de la relation entre l'élément en rouge et le reste de la 
phrase : relation de cause (C), de condition (Cond), de temps ( $\mathrm{T}$ ) ou autre (A, avec demande de précision).»

Pour finir, afin de mieux confronter les résultats de notre test 1 à la prédiction $\mathrm{P} 1$, nous avons demandé à un enseignant de norvégien de relever dans les traductions d'éventuels éléments peu ou pas acceptables selon les règles d'usage courant de la langue norvégienne.

\section{[4] ANALYSE}

\section{[4.1] Récapitulatif des résultats aux tests 1 et 2}

Un examen des résultats obtenus dans les tests 1 et 2, balisé par les prédictions et interrogations formulées plus haut, laisse apparaître une première typologie de stratégies de traductions - génératrices d'écueils interprétatifs. Deux types dominent cette catégorisation. On distingue ainsi les traductions issues de calques syntaxiques des autres, fruits d'un processus d'interprétation sémantique. Chacune de ces stratégies se décline sous diverses formes que nous décrirons maintenant, exemples d'étudiants (répertoriés de $a$ à $i$ ) à l'appui.

\section{[4.2] Le calque syntaxique}

Dans l'exercice de traduction (Test 2), 31\% des réponses résultent de transpositions linguistiques où la forme, la place et l'ordre des mots des énoncés sources semblent être les repères qui prévalent sur le sens et la qualité de l'expression en norvégien.

En dépit de l'indication associée à la consigne du test 2 - «Souvenez-vous : dans le doute, on ne traduit pas les mots, on traduit le sens !! » - on note un calque fréquent de la structure française du syntagme adjectival ou participial en $\mathrm{CD}$ antéposée. Cette reproduction est tantôt parfaitement tantôt partiellement superposable à la syntaxe source.

$11 \%$ des réponses données correspondent à des calques rigoureux des $\mathrm{CD}$ employées dans nos exemples français. Nous avions annoncé dans notre introduction les limites de telles constructions en norvégien. Concrètement, les occurrences du test où elles fonctionnent et demeurent syntaxiquement relativement acceptables sont rares. Les voici :

(13) a. Ferdigutdannet og slående vakker, reiste Jeanne seg for å holde en tale. $\{26 e\}$

b. Ungdom, hør godt etter denne eldre herrens vise ord! $\{27 i\}$

On soulignera ici que le norvégien employé est syntaxiquement défini comme 'relativement acceptable' par le référent norvégien. Or, malgré leurs défauts 
stylistiques, ces réponses purement calquées sur les textes sources sont admises en raison - jugeons-nous - d'un trait sémantique commun de la connexion sémantique exprimée en $\{26\}$ et $\{27\}$, relevé par le panel du test 1 comme purement et simplement "descriptif». L'apostrophe de l'item $\{27\}$ conforte cette idée : Jeunes gens ne sert qu'à préciser l'identité du sujet d'écoutez. Pour traduire cette entrée, 8 étudiants sur 9 ont choisi la $C D$ en position initiale. Par ailleurs, comme nous l'avions annoncé dans notre présentation théorique, $\{26 e\}$ confirme le fait que la $\mathrm{CD}$ fonctionne en norvégien dans des contextes pompeux. De même, un coup d'œil sur l'exemple $\{20\}$ consolide le postulat selon lequel la CD norvégienne est plus appropriée aux cas de connexions descriptives. Tandis que le panel du test 1 a classé la relation sémantique exprimée en $\{20\}$ comme conditionnelle, la traduction de la phrase par le recours à une $\mathrm{CD}$ est enregistrée comme non-acceptable.

*Varm, er denne suppa bra for turgåere. $\{20 d\}$

On en déduira que le message sémantique délivré dans le texte original exige une forme syntaxique plus explicite en norvégien.

Dans de nombreux autres cas, si les apprenants ont abandonné l'idée d'un transfert fidèle des syntagmes adjectivaux ou participiaux en CD, ils s'attachent toutefois à reproduire l'emphase par l'antéposition. On notera ici la récurrence des syntagmes prépositionnels ${ }^{4}$ introduits par « som » et placés en tête d'énoncé (20\% des réponses), en soulignant déjà les risques d'un tel emploi dont le poids sémantique et les contraintes syntaxiques sont probablement sous-estimés. Voyons sur pièces :

a. Som ung, giftet Dimitri seg med en indonesisk prinsesse. $\{5 b\}$

b. ??Som yngre enn sin kone, kunne ikke Dimitri forstå hvorfor hun var sliten. $\{8 c\}$

c. ??Som ferdigutdannet i industriell design i 1995, bor Jeanne nå i Paris. $\{25 e\}$

d. ??Som ferdigutdannet og vakker, gjorde Jeanne seg klar for å holde en tale. $\{26 c\}$

Les exemples $\{5 b\}$ et $\{8 c\}$ mettent en exergue la particularité syntaxique de "som ", incompatible avec un groupe adjectival comparatif yngre enn sin kone. D'autre part, comme le montrent les exemples $\{25 e\}$ et $\{26 c\}$, certains emplois du syntagme introduit par «som » confèrent un sens à la CD qui trahit la valeur

[4] Cette catégorisation est discutable. Nous avançons néanmoins qu'en raison de son usage particulier ici, som, plus traditionnellement qualifié comme « conjonction », prend une valeur prépositionnelle dans ces exemples. 
descriptive de la relation originale en établissant une relation de cause à effet entre la CD et la phrase matrice. En bref, «som » a des exigences syntaxiques et un sémantisme inhérent dont les étudiants doivent apprendre à se méfier.

Dans une volonté apparente de rester fidèles à la syntaxe des phrases originales, certaines traductions ont pris la forme de phrases elliptiques indépendantes, sans inversion du sujet mais avec un maintien thématique grâce à l'emphase en position initiale. On note ainsi avec intérêt que cette solution choisie en $\{15 c\}$ est appropriée, là où elle échoue avec une simple CD en $\{15 b\}$ :

(16) a. ??Ferdigutdannet eller ikke, så tilbyr vi Jeanne en interessant stilling i selskapet vårt. $\{15 b\}$

b. Ferdigutdannet eller ikke, vi tilbyr en interessant stilling til Jeanne i selskapet vårt. $\{15 c\}$

\section{[4.3] La saisie du sens des connexions}

Comme nous l'avons signalé dans la présentation théorique, nombre de $\mathrm{CD}$ françaises véhiculent des informations circonstancielles. Dans le processus de traduction, lorsque l'apprenant ne se laisse pas aveugler par la forme de l'énoncé mais s'attache à son sens, ses solutions de traduction norvégienne deviennent beaucoup plus explicites, et s'exposent aux risques du faux-sens. Toutefois, dans la plupart des items de notre test, la reformulation par une subordonnée ou par un syntagme prépositionnel tenant lieu de compléments circonstanciels était bienvenue, comme dans les exemples suivants :

(17) a. Da han var yngre hadde Dimitri liten tro på seg selv. $\{6 f\}$

b. Da hun var ferdigutdannet, bestemte Jeanne seg for å bli gründer. $\{14 g\}$

c. Ettersom den ble gjenoppbygget veldig raskt etter orkanen, mistet skolen sin arkitektoniske stil. \{18e\}

Notons que sur les 22 entrées qui ont été estimées comme exprimant des valeurs circonstancielles par le panel du test $1,34 \%$ des réponses du test 2 reflètent des valeurs circonstancielles équivalentes. Parmi ces valeurs, c'est celle de temps simultanéité ou consécution - qui apparaît le plus souvent (46\% des traductions exprimant du circonstanciel) et s'affirme comme le choix prioritaire des apprenants lorsque les français consultés dans le test 1 laissent une alternative ; ainsi dans l'exemple $\{12\}$, jugé initialement à la fois causal et temporel :

(18) a. Idet Jeanne fikk sin grad var hun rask med å fortelle sin mor nyheten. $\{12 g\}$ 
b. Etter å ha bestått eksamen var Jeanne rask med å fortelle nyheten til moren $\sin .\{12 d\}$

L'alternative d'options interprétatives fréquemment livrée dans le test 1 laisse transparaître l'ambiguïté des contenus sémantiques. Dans l'exemple $\{10\}$, le panel français a eu du mal à conclure sur la nature de la relation sémantique. De leur point de vue, il s'agissait soit d'un lien circonstanciel de cause (parce qu'elle était diplômée, Jeanne pensait...) soit de condition (sa mère serait fière d'elle si elle était diplômée). Dans sa traduction, l'étudiant h exprime une relation de temps dans une subordonnée circonstancielle ; ce qui est, en fait, également concevable :

(19) Jeanne trodde at moren hennes ville bli stolt av henne når hun var ferdigutdannet. $\{10 \mathrm{~h}\}$

De la gestion acceptable de l'ambigüité au faux-sens, il n'y a qu'un pas. Le fauxsens laisse apparaître un problème de saisie du texte source. En ce qui concerne la $\mathrm{CD}$, réservoir de non-dits, nombreuses sont les occasions de faire fausse route pour des apprenants de niveau B1.

(20) a. Da han var yngre ville Dimitri reise jord rundt. $\{9 f\}$

b. Etter utdannelsen tok Jeanne mer initiativ på jobben. $\{13 a\}$

On a en $\{9 f\}$ et $\{13 a\}$ des relations de temps qui passent à côté de l'essence même, conditionnelle, des messages initiaux. Dans l'ensemble du test 2 , seules $17 \%$ des entrées évaluées comme conditionnelles par les français du test 1 ont été saisies comme telles dans les traductions. Ceci suggère que la valeur conditionnelle, à laquelle une flexion morphologique du verbe contribue, échappe encore aux capacités d'interprétation des apprenants de niveau B1.

Enfin, comme le montrent les exemples suivants, nous retiendrons une tentative souvent réussie d'esquiver les dangers de l'ambigüité. Ceci apparaît dans les traductions par la subordonnée relative déterminative, avec un choix descriptif clair, inscrit dans une forme de neutralité acceptable $\{25 g\}$. Certaines reformulations se préservent encore plus de tout parti-pris. Parmi les réponses au test 2, vingt traductions réparties sur dix entrées donnent l'impression d'une simple coordination pour laquelle on ne saurait dire si elle s'apparente à la qualification pure d'un des arguments de la phrase matrice - le sujet en général - ou si elle suggère, entre les clauses, un rapport de cause, de simultanéité ou de consécution. Bien que peu d'information soit véhiculée ainsi, voire même nettement moins que ce que le texte source transmet, on reste à l'écart de toute faute de sens et de forme par un choix syntaxique simple et sûr $\{20 f\}$. 
a. Jeanne, som tok en grad i industriell design i 1995, bor nå i Paris. $\{25 g\}$

b. Denne varme tomatsuppa vil gjøre godt for turgåere. $\{20 f\}$

\section{[4.4] Le défi de la co-référence}

Notre prédiction $\mathrm{P} 3$ portait sur la difficulté à identifier le référent de la $\mathrm{CD}$ dans les situations $\{15\},\{19\}$ et $\{27\}$ où le sujet n'est pas clairement exprimé dans la phrase matrice. Les phrases $\{19\}$ et $\{27\}$ n'ont pas posé de problème qu'on puisse associer au défi co-référenciel. En revanche, on relèvera la difficulté pour $\{15\}$. La phrase originale mettait en scène une co-indexation de l'objet datif de la phrase matrice. Pour traduire ceci, les apprenants qui ne se sont pas concentrés en priorité sur le rapport circonstanciel de concession (Qu'elle fût diplômée ou non), soit 4 apprenants sur 9, ont fourni des solutions syntaxiquement incorrectes pour des raisons de référence, comme ici :

(22) Vitnemål eller ikke, tilbyr vi Jeanne en spennende stilling i vårt firma. $\{15 d\}$

\section{[4.5] Bilan}

La façon dont les apprenants de notre test 2 font face au défi de l'identification référentielle reflète les stratégies mises en œuvre dans le processus de traduction. Nous avons introduit notre analyse en en soulignant les deux grands axes : le calque syntaxique et l'interprétation sémantique. Bien que nous ayons catégorisé nos résultats par souci de clarté, il est évident que ces deux stratégies ne sont pas, ni ne doivent être incompatibles. Toutefois, la récurrence de traductions calquées qui ne laissent pas apparaître de prise de position sémantique réelle amène à penser que le calque syntaxique résulte souvent d'un simple défaut d'apprenti traducteur aveuglé par la forme du texte source et, peut-être, d'une peur de l'engagement interprétatif. La saisie d'une connexion descriptive est ainsi largement privilégiée par les étudiants (dans 23 items sur 30) quand seuls 9 items sur 30 bénéficient de cette étiquette au terme du test 1 .

Du présent travail ressort, par ailleurs, la différence d'emplois des syntagmes adjectivaux et participiaux en CD en français et en norvégien. On a vu que l'apprenant avait tendance à réduire le bagage sémantique de la $\mathrm{CD}$ française à la description pure du constituant auquel elle référait dans la phrase matrice. Si notre étude laisse entendre que la $\mathrm{CD}$ de ce type en norvégien demeure relativement limitée à une fonction descriptive, elle signale en substance l'importance des impacts syntaxico-sémantiques sur la qualité d'interprétation, et de traduction de constructions finalement assez courantes. Premièrement, on retiendra que les marges d'acceptabilité syntaxique d'un calque norvégien de la $\mathrm{CD}$ 
française sont réduites. Deuxièmement, on rappellera que la $C D$ française a un bagage sémantique largement plus conséquent que la norvégienne.

\section{[5] CONCLUSION}

Notre présentation syntaxico-sémantique des syntagmes adjectivaux et participiaux en $C D$ françaises a jeté les fondations pour un travail empirique et contrastif concernant les choix de traduction des apprenants norvégiens L2.

Dans le cadre de la méthode d'action-recherche que nous avons menée, en guise de bilan et de conclusion, le retour didactique aux étudiants, empreint des résultats de nos analyses, prendra la forme d'une hiérarchie qualitative, en termes syntaxico-sémantiques, des types de traductions des syntagmes adjectivaux et participiaux en structures détachées. La voici :

\begin{tabular}{|c|c|c|c|c|c|c|}
\hline & & \multicolumn{5}{|c|}{ Types de traductions norvégiennes } \\
\hline & & $\begin{array}{l}\text { Calque } \\
\text { parfait }\end{array}$ & $\begin{array}{c}\text { Groupe } \\
\text { préposi- } \\
\text { tionnel } \\
\text { introduit } \\
\text { par } \\
\text { «som » }\end{array}$ & $\begin{array}{l}\text { Subor- } \\
\text { donnée } \\
\text { relative }\end{array}$ & $\begin{array}{l}\text { Subor- } \\
\text { donnée } \\
\text { circons- } \\
\text { tancielle }\end{array}$ & $\begin{array}{c}\text { Restructu- } \\
\text { ration to- } \\
\text { tale de la } \\
\text { phrase }\end{array}$ \\
\hline 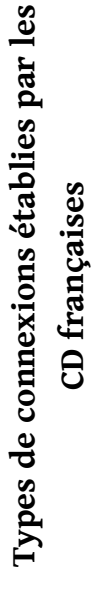 & $\begin{array}{c}\text { Connexion } \\
\text { descriptive } \\
\text { Connexion } \\
\text { descriptive } \\
\text { et/ou cir- } \\
\text { constan- } \\
\text { cielle } \\
\text { Connexion } \\
\text { circons- } \\
\text { tancielle }\end{array}$ & $\begin{array}{l}X\{24 x\} \\
X\{8 z\}\end{array}$ & $\mathrm{X}$ & $X\{24 y\}$ & $X\{6 y\}$ & $\mathrm{X}$ \\
\hline
\end{tabular}

TABLEAU 1. TRADUCTIONS POSSIBLES DE LA CD FRANÇAISE

Le tableau 1 présente un récapitulatif des traductions possibles de la CD française. Il renvoie bien sûr à tous les commentaires et réserves mentionnés dans l'article. Au risque d'être taxé de rigidité prescriptive, il nourrit des visées 
didactiques. Il se base en conséquence sur les résultats de nos analyses et propose quelques simples directions, dont les meilleures sont en caractères gras, suppléées de quelques exemples notés entre parenthèses en annonce des commentaires supplémentaires ci-dessous.

Dans l'exemple $\{8\}$ de nos tests, la CD plus jeune que son épouse établit une relation descriptive avec le sujet Dimitri et circonstancielle avec l'ensemble de la clause matrice. Dans ce cas, deux choix de traductions se détachent du lot; le premier, dépourvu de risque interprétatif, est celui de la subordonnée relative non-restrictive, le second résulte d'une interprétation circonstancielle de cause.

(23) Plus jeune que son épouse, Dimitri ne comprenait pas pourquoi sa femme était si souvent fatiguée. $\{8\}$

$\rightarrow$ Dimitri, som var yngre enn sin kone, hadde problemer med å forstå hvorfor hun var så ofte sliten. $\{8 x\}$

$\rightarrow$ Ettersom/siden han var yngre enn sin kone, hadde Dimitri problemer med å forstå hvorfor hun var så ofte sliten. $\{8 y\}$

Une traduction directe de la $\mathrm{CD}$ française n'est pas acceptable en norvégien :

(24) $\rightarrow$ ??Yngre enn sin kone forsto ikke Dimitri hvorfor hun var så ofte sliten. $\{8 z\}$

La CD « plus jeune » de l'exemple $\{6\}$ construit un lien qu'on ne peut interpréter qu'en relation circonstancielle avec la phrase matrice. Pour cette raison, les seules traductions qui nous semblent envisageables excluent le recours à une $C D$ et privilégieront l'emploi de la subordonnée circonstancielle :

(25) Plus jeune, Dimitri avait peu confiance en lui. $\{6\}$

$\rightarrow$ ??Yngre hadde Dimitri lav selvtillit. $\{6 x\}$

$\rightarrow$ Da han var yngre, hadde Dimitri lav selvtillit. $\{6 y\}$

Enfin, malgré le non-respect de la position initiale qui engendre une rupture thématique, le choix de la subordonnée relative non-restrictive dans les situations exclusivement descriptives demeure le plus sûr. Comme on l'a vu, le calque parfait et la construction introduite par " som » sont soumis à une kyrielle de contraintes syntaxico-sémantiques, autant de dangers pour la correction du produit final en l'absence de contexte stylistique précis. En voici l'illustration :

(26) Diplômée en dessin industriel depuis 1995, Jeanne habite maintenant à Paris. $\{25\}$

$\rightarrow$ ?Ferdigutdannet $\mathrm{i}$ industriell design siden 1995, bor Jeanne nå 


\section{i Paris. $\{25 x\}$}

$\rightarrow$ Jeanne, som ble ferdigutdannet $\mathrm{i}$ industriell design i 1995, bor nå i Paris. $\{25 y\}$

Ce tableau récapitulatif des possibles traductions des syntagmes adjectivaux et participiaux est conçu à l'intention d'apprenants B1. Il ne tient ainsi pas compte de la portée sémantique de la $\mathrm{CD}$, dimension essentielle à la compréhension de la construction discursive, que nous avons toutefois jugée prématurée pour atteindre des objectifs immédiats de typologie hiérarchisée des traductions de la CD. Sa mise en évidence des différences syntaxico-sémantiques entre le français et le norvégien offre, entre autres, des pistes de réflexion sur l'expression des relations logiques et circonstancielles dans les deux langue et s'inscrit dans la lignée des travaux de Hans Petter Helland sur les rapprochements entre le norvégien et le français.

Merci à toi, Hans Petter, pour ta talentueuse et généreuse contribution à nos formations et nos carrières !

\section{BIBLIOGRAPHIE}

Aleksandrova, Angelina. 2015. La portée cadrative des constructions détachées : l'exemple des portraits journalistiques, Studii de lingvistică 5. 73-90.

Chomsky, Noam. 1981. Lectures on government and binding. Berlin : Mouton.

Combettes, Bernard. 2005. Les constructions détachées comme cadres de discours, Langue française 158(4). 31-44.

Combettes, Bernard. 1998, Thématisation, topicalisation, et éléments nonréférentiels : le cas de l'adjectif détaché, Cahiers de praxématique 30. 133-159.

Demeuse, Marc. et al., 2005, La fiabilité de l'évaluation des compétences linguistiques pour des adultes non francophones : présentation d'un protocole d'évaluation $<$ https://www.lefrancaisdesaffaires.fr/wpcontent/uploads/2016/05/3.TEF-Admee2005-Fiabilit\%C3\%A9.pdf> (consulté le 4 janvier 2021)

Havu, Eva \& Pierrard, Michel. 2015. Les participiales absolues sont-elles des subordonnées circonstancielles? A propos de la sous-spécifications des rapports syntaxo-sémantique, SHS Web of Conferences 46. Les Ulis : EDP Sciences

Helland, Hans Petter. 2006/2014. Ny fransk grammatikk. Syntaks, morfologi 
og semantikk. Oslo: Universitetsforlaget.

Helland, Hans Petter. 2013. Non-finite adjuncts in French. Dans T. Lohndal (ed.), In Search of Universal Grammar: From Old Norse to Zoque, 117-136. Amsterdam: John Benjamins.

Helland, Hans Petter \& Hobæk-Haff, Marianne. 2000. Nominalfrasen og dens satelitter. Romansk Forum 12.

Hobæk Haff, Marianne. 2012. On absolutes in French, German and Norwegian. Dans C. Fabricius-Hansen D. Haug (eds.), Big Events, Small Clauses: The Grammar of Elaboration, 259-286. Berlin: Walter de Gruyter.

Puren, Christian. 2013. Mettre en œuvre ses méthodes de recherche, $<$ www.christianpuren.com/coursméthodologie-de-la-recherche-endlc/chapitre-5-mettre-en-oeuvre-ses-méthodes-derecherche/>, 23-29. (consulté le 18 décembre 2020)

\section{ANNEXE : TEST}

Voici, non randomisées, les entrées des tests 1 et 2 :

\{1\} Tout jeune garçon parmi les grands, Pierre n'avait pas souvent le droit de décider dans la cour de récréation.

\{2\} Tout jeune garçon, Pierre n'aurait pas pu quitter ses parents pendant plus d'une semaine.

\{3\} Tout jeune garçon, Pierre était calme.

44) Plus jeune, Dimitri aurait demandé sa voisine en mariage.

\{5\} Plus jeune, Dimitri avait épousé une princesse indonésienne.

\{6\} Plus jeune, Dimitri avait peu confiance en lui.

\{7\} Plus jeune que son épouse, Dimitri avait du mal à se faire respecter par sa belle-famille.

\{8\} Plus jeune que son épouse, Dimitri ne comprenait pas pourquoi sa femme était si souvent fatiguée.

\{9\} Plus jeune, Dimitri serait parti faire le tour du monde.

\{10\} Diplômée, Jeanne pensait que sa mère serait enfin fière d'elle.

\{11\} Diplômée, Jeanne n'aurait pas hésiter à postuler comme directrice.

\{12\} Diplômée, Jeanne s'est empressée d'annoncer la nouvelle à sa mère.

\{13\} Diplômée, Jeanne prendrait plus d'initiative au travail.

\{14\} Diplômée, Jeanne a décidé de monter sa propre entreprise.

\{15\} Diplômée ou non, nous offrons à Jeanne un poste intéressant dans notre entreprise. 
\{16\} Reconstruite après l'ouragan, l'école aurait pu aussi servir de centre d'accueil pour les réfugiés.

\{17\} Reconstruite après l'ouragan, l'école a ouvert ses portes aux enfants des familles démunies.

\{18\} Reconstruite très rapidement après l'ouragan, l'école a perdu son style architectural.

\{19\} Enfant ou adulte, chacun à sa manière pourra apprécier le spectacle.

\{20\} Chaude, cette soupe à la tomate ferait du bien aux randonneurs.

\{21\} Chaude, cette soupe à la tomate est bien meilleure.

\{22\} Trop chaude, cette soupe à la tomate risque de déplaire au bébé.

\{23\} Reconstruites en briques ou en bois, les écoles de la ville sont assez grandes pour accueillir la plupart des enfants.

\{24\} Reconstruite dans un style moderne, l'école est au centre du village.

\{25\} Diplômée en dessin industriel depuis 1995, Jeanne habite mainte nant à Paris.

\{26\} Diplômée et resplendissante, Jeanne s'est levée pour faire un discours.

\{27\} Jeunes gens, écoutez-bien les paroles sages de ce vieil homme!

\{28\} Pierre aimait jouer avec les enfants de ses voisins.

\{29\} Avec les enfants du village, Pierre a construit un château de sable impressionnant.

\{30\} Je ne savais pas qu'il était plus jeune que sa femme.

COORDONNÉES

Nelly Foucher Stenkløv

NTNU

nelly.stenklov@ntnu.no

Eirik Hvidsten

NTNU

eirik.hvidsten@ntnu.no 